\title{
Morphological and molecular characterization of fungal pathogen, Magnaphorthe oryzae
}

\begin{abstract}
Rice is arguably the most crucial food crops supplying quarter of calories intake. Fungal pathogen, Magnaphorthe oryzae promotes blast disease unconditionally to gramineous host including rice species. This disease spurred an outbreaks and constant threat to cereal production. Global rice yield declining almost 10-30\% including Malaysia. As Magnaphorthe oryzae and its host is model in disease plant study, the rice blast pathosystem has been the subject of intense interest to overcome the importance of the disease to world agriculture. Therefore, in this study, our prime objective was to isolate samples of Magnaphorthe oryzae from diseased leaf obtained from MARDI Seberang Perai, Penang, Malaysia. Molecular identification was performed by sequences analysis from internal transcribed spacer (ITS) region of nuclear ribosomal RNA genes. Phylogenetic affiliation of the isolated samples were analyzed by comparing the ITS sequences with those deposited in the GenBank database. The sequence of the isolate demonstrated at least $99 \%$ nucleotide identity with the corresponding sequence in GenBank for Magnaphorthe oryzae. Morphological observed under microscope demonstrated that the structure of conidia followed similar characteristic as M. oryzae. Finding in this study provide useful information for breeding programs, epidemiology studies and improved disease management.
\end{abstract}

Keyword: Magnaphorthe oryzae; Fungal pathogen; Molecular characterization; Morphological 